\title{
Generation flow in field theory and strings
}

\author{
Saúl Ramos-Sánchez, ${ }^{a}$ Michael Ratz, ${ }^{b}$ Yuri Shirman, ${ }^{b}$ Shreya Shukla ${ }^{b}$ and \\ Michael Waterbury ${ }^{b}$ \\ ${ }^{a}$ Instituto de Física, Universidad Nacional Autónoma de México, \\ POB 20-364, Cd.Mx. 01000, México \\ ${ }^{b}$ Department of Physics and Astronomy, University of California, \\ Irvine, CA 92697-4575 U.S.A. \\ E-mail: ramos@fisica.unam.mx, mratz@uci.edu, yshirman@uci.edu, \\ sshukla4@uci.edu, mwaterbu@uci.edu
}

ABSTRACT: Nontrivial strong dynamics often leads to the appearance of chiral composites. In phenomenological applications, these can either play the role of Standard Model particles or lift chiral exotics by partnering with them in mass terms. As a consequence, the RG flow may change the effective number of chiral generations, a phenomenon we call generation flow. We provide explicit constructions of globally consistent string models exhibiting generation flow. Since such constructions were misclassified in the traditional model searches, our results imply that more care than usually appreciated has to be taken when scanning string compactifications for realistic models.

KEywords: Compactification and String Models, Confinement, Nonperturbative Effects, Superstring Vacua

ArXiv ePrint: 2109.01681 


\section{Contents}

1 Introduction 1

$2 s$-confinement and gapped chiral fermions $\quad 3$

$2.1 s$-confining $\mathrm{SU}(2)_{s}$ model 3

2.2 Mass gap model 4

3 Generation flows in GUTs 5

$3.14 \rightsquigarrow 3$ generation flow 6

$\begin{array}{ll}3.2 & 2 \rightsquigarrow 3 \text { generation flow }\end{array}$

4 Generation flow in string models $\quad 9$

$\begin{array}{ll}4.1 \text { Model scan } & 10\end{array}$

$\begin{array}{lll}4.2 & \text { Models } & 11\end{array}$

$\begin{array}{lll}4.3 & \text { Discussion } & 12\end{array}$

$\begin{array}{lll}5 & \text { Summary } & 13\end{array}$

$\begin{array}{ll}\text { A Orbifold model definitions } & 14\end{array}$

A.1 Details of the $4 \rightsquigarrow 3$ heterotic orbifold model 14

A.2 Details of the $2 \rightsquigarrow 3$ heterotic orbifold model 15

\section{Introduction}

One of the curious features of the Standard Model (SM) of particle physics is the repetition of families. That is, the matter content of the SM comprises three copies of fermions carrying identical SM gauge quantum numbers. While the number of generations is generally arbitrary in field theoretic extensions of the SM, such as a Grand Unified Theory (GUT), in string theory it can be thought of as a prediction of any specific model or compactification. Hence, the number of generations is often used as one of the first selection filters applied in a search for promising string models. It is the purpose of this study to point out that non-perturbative field theoretic dynamics may modify the number of effective generations in the process of renormalization group ( $R G$ ) flow. Thus, some additional care is required when counting the number of generations in candidates for ultraviolet (UV) completions of the SM, in particular in string models.

In this paper, we will concentrate on supersymmetric models both because it is convenient in the context of string model building and because the relevant non-perturbative dynamics is under qualitative and often quantitative control in such theories. As shown by Seiberg [1], non-perturbative effects can have a dramatic impact on gauge theories. In particular, due to confinement and duality, the degrees of freedom appropriate for describing 
infrared (IR) physics often differ considerably from the UV degrees of freedom. Throughout this paper, aiming at preserving the chirality of the SM (or its GUT completion), we consider confinement without chiral symmetry breaking (so-called $s$-confinement $[2,3]$ ). Since the low-energy degrees of freedom in these models are composites of the elementary fields, they usually transform in different representations of the unbroken global symmetry. When a subgroup of such global symmetry is identified with a GUT or the SM gauge group, a new, composite, chiral generation may emerge in the IR or, alternatively, an existing chiral generation may become massive. The first of these phenomena was initially used in $[4,5]$ to construct realistic extensions of the minimal supersymmetric Standard Model with some of the third generation quarks and Higgs bosons arising as composites of strong dynamics. In this approach, which we will refer to as the Nelson-Strassler (NS) mechanism, the RG flow leads to the appearance of light chiral composites in the IR thus increasing the effective number of chiral generations. The NS mechanism may be modified in several fairly obvious ways. For example, some of the composites may acquire masses by mixing with elementary chiral fields, modifying the spectrum of light fields in the IR in nontrivial ways. When all of the composites acquire mass, the model is in the second regime which attracted attention more recently [6]. We will refer to the second phenomenon as the Razamat-Tong (RT) mechanism. Here all of the composites of strong dynamics acquire masses by partnering with elementary degrees of freedom and thus reduce the number of effective generations in the IR. As we will argue, these two mechanisms can be continuously connected by introducing mass terms for vector-like elementary fields, which are allowed to mix with the composites. When masses of vector-like fields are small while the mixing between elementary fields and composites is of order one, the theory flows to the RT limit where all the light fields are elementary. On the other hand, in the limit of large mass the vector-like elementary fields decouple, leaving massless composites behind. In this case, the theory flows to the NS limit where some light fields are composites. By varying the mass terms, one can interpolate between the two limits, and for intermediate values of the mass term some IR degrees of freedom will be partially composite. Furthermore, one has freedom to decouple any number of composites. In general, however, non-perturbative dynamics affects RG flow and modifies the effective number of chiral generations in the IR. We will refer to these phenomena as generation flow.

It is then natural to ask whether generation flow can occur in scenarios where the number of generations is predicted from other data. This is particularly relevant for string model building (cf. e.g. [7] for a review), where one obtains the SM generations from string compactifications. We will argue that generation flow indeed occurs in some globally consistent string models. In these constructions, the true number of generations in the IR description can differ from the tree-level value that one obtains at the compactification scale. Hence, a search for 3-generation models in string theory has to go beyond the tree-level analysis.

This paper is organized as follows. In section 2, we will review the RT mechanism of gapped chiral fermions. In section 3 , we construct models exhibiting generation flow towards a 3-generation theory with (a GUT completion of) the SM gauge group in the IR. Our first example is a $4 \rightsquigarrow 3$ model based on the RT mechanism where all the IR degrees of freedom are elementary. We then construct a generalization of the $4 \rightsquigarrow 3$ model where some 
of the third generation fields are composite. We point out that our construction is analogous to the NS mechanism $[4,5]$. This motivates us to build a $2 \rightsquigarrow 3$ model with an upward generation flow. Furthermore, we discuss the stability of the chirally symmetric vacua in $s$-confining models under the deformations which induce generation flow. While such deformations may generally destabilize the vacua by non-perturbative dynamics (see [8] for a more detailed discussion), we argue that the chirally symmetric vacua survive in our models. In section 4 , we collect evidence for the existence of string models exhibiting generation flow by presenting explicit examples. Finally, section 5 contains our conclusions.

\section{$2 s$-confinement and gapped chiral fermions}

We begin by briefly reviewing dynamics of supersymmetric gapped fermion models introduced in [6]. In the following we will take the approach of [8] to building models of chiral gapped fermions. This approach starts with SUSY QCD models that exhibit confinement without chiral symmetry breaking on smooth moduli space [1]. ${ }^{1}$ For our purposes it is convenient to restrict attention to $s$-confinement in SU(2) $s$ SUSY QCD with six chiral doublet superfields and thus $\mathrm{SU}(6)$ chiral global symmetry. We review the dynamics of this model in the subsection 2.1. In the subsection 2.2, we discuss the deformation of the SUSY QCD required to arrive at mass gap models of [6].

\section{$2.1 s$-confining $\mathrm{SU}(2)_{s}$ model}

The model outlined above possesses $\mathrm{SU}(6) \times \mathrm{SU}(2)_{s}$ symmetry, where $\mathrm{SU}(6)$ is a chiral global symmetry while $\mathrm{SU}(2)_{s}$ is a strongly interacting $s$-confining gauge group. For future convenience we will assign quark superfields to $(\overline{\mathbf{6}}, \mathbf{2})$ representation of the symmetry group. The theory possesses a set of classical $D$-flat directions which can be parameterized either in terms of squark vacuum expectation values (VEVs) or in terms of gauge invariant mesons which are classically defined as $M_{i j} \sim Q_{i} Q_{j} / \Lambda$, where we suppressed contraction of $\mathrm{SU}(2)_{s^{-}}$ color indices and the dynamical scale of the quantum theory $\Lambda$ is introduced on dimensional grounds. The mesons $M$ transform in the conjugate antisymmetric representation of the global SU(6) symmetry $\overline{\mathbf{1 5}}$. However, since quark VEVs satisfy a set of algebraic identities, not all meson VEVs are independent. These classical constraints imply a set of relations between the mesons,

$$
\varepsilon^{i_{1} \ldots i_{6}} M_{i_{3} i_{4}} M_{i_{5} i_{6}}=0 .
$$

One may implement these constraints in the composite description of the theory by postulating a dynamical superpotential

$$
\mathscr{W}_{s}=\varepsilon^{i_{1} \ldots i_{6}} M_{i_{1} i_{2}} M_{i_{3} i_{4}} M_{i_{5} i_{6}} \equiv \operatorname{Pf}(M) .
$$

The moduli space parameterized by mesons $M$ together with the superpotential $(2.2)$ coincides with the classical moduli space of the theory parameterized by quark VEVs satisfying $D$-flatness conditions. It was shown in [1] that the classical moduli space of

\footnotetext{
${ }^{1}$ This dynamics is usually referred to as $s$-confinement. See [3] for a complete classification of such theories.
} 
vacua remains unmodified quantum mechanically and the IR physics is described in terms of weakly interacting mesons with the superpotential (2.2). While the chiral global symmetry of this model is broken at a generic point on the moduli space, the chiral symmetry remains unbroken at the origin where the theory exhibits confinement without chiral symmetry breaking. This is precisely the vacuum we are interested in.

\subsection{Mass gap model}

For phenomenological purposes we are interested in gauging $\mathrm{SU}(6)$ global symmetry of the $s$-confining model discussed in the previous subsection (more precisely we are interested in gauging a subgroup of $\mathrm{SU}(6)$, such as a $\mathrm{GUT} \mathrm{SU}(5)$ or the $\mathrm{SM}$ group $\mathrm{SU}(3) \times \mathrm{SU}(2) \times \mathrm{U}(1))$. To this end, one must introduce a set of spectator fields charged under $\mathrm{SU}(6)$ but not $\mathrm{SU}(2)_{s}$ (so that the $s$-confining dynamics remains unaffected) to ensure a cancellation of the cubic $\mathrm{SU}(6)$ anomaly. This can be achieved, for example, by introducing spectators that transform in representations of $\mathrm{SU}(6)$ conjugate to those of elementary fields, i.e. by adding two spectators with quantum numbers given by $(\mathbf{6}, \mathbf{1})$. Alternatively, one can introduce a single spectator $S$ in an $\mathrm{SU}(6)$ representation conjugate to the one of the mesons, i.e. transforming as $(\mathbf{1 5}, \mathbf{1})$. In the former case, the theory remains chiral both in the UV and IR. This is because $\mathrm{SU}(2)_{s}$ is not yet confined in the UV and the matter fields transform in chiral representations of the full $\mathrm{SU}(6) \times \mathrm{SU}(2)_{s}$ symmetry, while the representations of IR degrees of freedom are chiral under $\mathrm{SU}(6)$. However, in the latter case, the chiral properties of the model change as the theory flows from the UV to the IR. While the UV theory is clearly chiral, the IR degrees of freedom, the mesons $M$ and spectators $S$, transform in conjugate representations and thus form a single vector-like representation. By choosing to cancel anomalies with the spectator $S$ in the antisymmetric representation, we will be able to construct a model flows from a gapless, chiral phase in the UV to a gapped phase in the IR.

Since the matter content in the IR is non-chiral, a mass term, $S M$, is allowed in the IR superpotential. In terms of the UV degrees of freedom, this mass term corresponds to a marginal operator, $S Q^{2}$. Thus, we deform the $s$-confining model by a tree-level superpotential

$$
\mathscr{W}=y S Q^{2}=c \Lambda S M
$$

where the numerical coefficient $c$ represents both an arbitrary Yukawa coupling $y$ of the UV theory and the fact that the mass scale generated by confinement is not directly calculable.

At this point one might be tempted to conclude that a mass gap develops in the chirally symmetric vacuum at the origin, while the rest of the moduli space is lifted by the equations of motion for $S$ and $M$. However, while ultimately correct, this conclusion is somewhat premature. Indeed, while lifting $\mathrm{SU}(2)_{s}$ D-flat directions, the deformation (2.3) introduces new classical flat directions, those parameterized by $\mathrm{SU}(2)_{s}$ singlets $S$. Since any VEVs for $S$ would break the chiral symmetry, it is important to verify that the nonperturbative dynamical superpotential (2.2) does not destabilize these directions. A careful analysis [8] of the full superpotential in (2.2) and (2.3) demonstrates that $\mathrm{SU}(2)_{s}$ dynamics 
generates an effective superpotential for gauge singlets $S$ stabilizing them at the origin. ${ }^{2}$ While referring the reader to [8] for the full analysis, we present a simple argument here. Consider the theory at large $S$ where all quark superfields become heavy. In this region of the moduli space the low-energy physics is described in terms of a pure super-YangMills (SYM) SU $(2)_{s}$ theory with dynamical scale given by $\Lambda_{L}^{6}=\operatorname{Pf}(S) \Lambda^{3}$. The dynamics of the low-energy SYM in turn generates a gaugino condensate implying the existence of an effective superpotential

$$
\mathscr{W}_{\text {dyn }}=\Lambda_{L}^{3}=\left(\Lambda^{3} \operatorname{Pf}(S)\right)^{1 / 2}
$$

It is easy to see that this superpotential stabilizes $S$ near the origin.

The main lesson we learn from this example is a possibility that the RG flow may change the chiral properties of the theory and, in particular, may change the number of chiral generations. Here we define a chiral generation as a field transforming in an antisymmetric representation of the chiral symmetry accompanied by an appropriate number of fields in an antifundamental representation as required by anomaly cancellation conditions. Then the net number of generations is given by a difference between number of fields in an antisymmetric representation and in a conjugate antisymmetric representation, $\nu=n_{\text {日 }}-$ $n_{\overline{\mathrm{E}}}$. For example, in our example with $\mathrm{SU}(6)$ chiral symmetry the number of generations is given by $n_{\mathbf{1 5}}-n_{\overline{\mathbf{1 5}}}$. This definition is chosen such that it can be used throughout this study, and coincides with what one calls a generation in SU(5) GUTs. From the SU(6) perspective, our UV model is a one-generation model containing an antisymmetric, 15, and two antifundamental, $\overline{\mathbf{6}}$, of SU(6). On the other hand, the IR theory has no massless chiral superfields even while the chiral symmetry remains unbroken.

While the construction of [6] decreases the number of chiral generations in the IR, we will show in the following section that non-perturbative dynamics may also lead to an increase in the number of chiral generations. As we will see, the existence of generation flow offers immense opportunities for model building both in field theory (section 3) and string theory (section 4).

\section{Generation flows in GUTs}

The supersymmetric gapped fermion model reviewed in the previous section is based on an $\mathrm{SU}(2)_{s} s$-confining theory with $\mathrm{SU}(6)$ global symmetry. Generalizations to $s$-confining $\mathrm{Sp}(2 N)$ with $\mathrm{SU}(2 N+4)$ global symmetry are straightforward $[6] .^{3}$ However, for phenomenological purposes one is interested in similar models with $\mathrm{SU}(5)$ or $\mathrm{SU}(3) \times \mathrm{SU}(2) \times$ $\mathrm{U}(1)$ global symmetry which can then be identified with the GUT or the SM gauge group. As shown in [6], this can be easily achieved simply by considering the model of section 2.2 and identifying GUT or SM gauge group with an appropriate subgroup of SU(6).

For example, to construct a one-generation $\mathrm{SU}(5) \times \mathrm{SU}(2)_{s}$ theory which behaves as a pure SYM SU(5) in the IR, one decomposes elementary fields of the model under SU(5)

\footnotetext{
${ }^{2}$ We stress that this conclusion is model dependent, and there exist models where the $S=0$ vacuum at the origin is destabilized, resulting in chiral symmetry breaking.

${ }^{3}$ See also discussion in [8].
} 
as follows

$$
S:(\mathbf{1 5}, \mathbf{1}) \rightarrow T:(\mathbf{1 0}, \mathbf{1}) \oplus F:(\mathbf{5}, \mathbf{1}), \quad Q:(\overline{\mathbf{6}}, \mathbf{2}) \rightarrow \bar{F}^{\prime}:(\overline{\mathbf{5}}, \mathbf{2}) \oplus \phi:(\mathbf{1}, \mathbf{2}) .
$$

The tree-level superpotential (2.3) and dynamical superpotential (2.2) can be easily written in the SU(5) language. One can verify that the UV description corresponds to a one-generation model complemented by a single vector-like flavor in a fundamental representation. As we learned in section 2, the $s$-confining dynamics leads to a unique ground state with an unbroken chiral symmetry and no light matter fields.

We are now ready to generalize the mass gap construction of RT [6] to obtain models where the number of chiral generations is changed through renormalization group flow but remains nonzero both in the UV and the IR. As we will see shortly, the RG flow may lead both to an increase and a decrease in the effective number of chiral generations. The latter can be achieved in two ways. In the first approach, as in the model of section 2 , some of the chiral elementary fields acquire masses by partnering with the chiral composites generated by confining dynamics. As a result, all the massless degrees of freedom in the IR are elementary fields of the theory. Just like in the model of section 2, the chirally symmetric vacuum is a unique ground state of this theory. The second approach is reminiscent of the construction first introduced in $[4,5]$. In this approach, some of the massless fields in the IR are composites even as other composites may become massive. Generically, models in this class retain the quantum moduli space and only one vacuum on this moduli space is chirally symmetric. Since IR degrees of freedom, including the massless composites, are to be identified with the SM multiplets, the motion along this moduli space is equivalent to motion along $D$-flat directions of a GUT or the SM. Note that the mechanism utilized in the second approach may also lead to an increase in the effective number of generations.

\section{$3.14 \rightsquigarrow 3$ generation flow}

We can now detail our general observations by building an explicit model of downward generation flow. Let us start with a more straightforward example, where the number of chiral generations decreases in the IR while all the composites are heavy. In particular, we construct a $4 \rightsquigarrow 3$ model, i.e. a model containing 4 generations in the UV and 3 generations in the IR. The matter fields of the model and their quantum numbers are presented in table 1a. Note that this matter content comprises the fields appearing in (3.1) complemented by three chiral flavors of SU(5) i.e. three copies of $T \oplus \bar{F}$. Thus, this is a four-generation model. It is easy to see that $\mathrm{SU}(2)_{s}$ dynamics is not affected by the introduction of additional chiral multiplets as long as one linear combination of the $T_{i}$ 's has the Yukawa coupling with $\bar{F}^{\prime}$ and $\phi$ that is implied by the superpotential (2.3). Indeed, at low energies $\mathrm{SU}(2)_{s}$ charged fields confine into $\overline{\mathcal{T}} \sim \bar{F}^{\prime} \bar{F}^{\prime} / \Lambda$ and $\overline{\mathcal{F}} \sim \bar{F}^{\prime} \phi / \Lambda$. The transformation properties of the IR degrees of freedom are given in table 1b. Finally, in the IR the superpotential (2.3) behaves like a mass term pairing composites $\overline{\mathcal{F}}$ and $\overline{\mathcal{T}}$ with $F$ and one copy of $T$, respectively. Repeating the analysis of section 2.2 one concludes that the classical flat directions parameterized by $F$ and $T$ are stabilized non-perturbatively.

Let us consider a generalization by noting that the symmetries of the model allow a mass term for the vector-like pair $F \oplus \bar{F}$. With this mass term, the full UV superpotential 


\begin{tabular}{ccl}
\hline$\#$ & irrep & label \\
\hline 4 & $(\mathbf{1 0}, \mathbf{1})$ & $T$ \\
2 & $(\overline{\mathbf{5}}, \mathbf{1})$ & $\bar{F}$ \\
\hline 1 & $(\overline{\mathbf{5}}, \mathbf{2})$ & $\bar{F}^{\prime}$ \\
1 & $(\mathbf{1}, \mathbf{2})$ & $\phi$ \\
\hline 1 & $(\mathbf{5}, \mathbf{1})$ & $F$ \\
1 & $(\overline{\mathbf{5}}, \mathbf{1})$ & $\bar{F}$ \\
\hline
\end{tabular}

(a) Unconfined spectrum.

\begin{tabular}{ccl}
\hline$\#$ & irrep & label \\
\hline 4 & $(\mathbf{1 0}, \mathbf{1})$ & $T$ \\
4 & $(\overline{\mathbf{5}}, \mathbf{1})$ & $\bar{F}, \overline{\mathcal{F}}$ \\
\hline 1 & $(\overline{\mathbf{1 0}}, \mathbf{1})$ & $\overline{\mathcal{T}}$ \\
1 & $(\mathbf{5}, \mathbf{1})$ & $F$ \\
\hline
\end{tabular}

(b) Confined spectrum.

Table 1. Summary of the $\mathrm{SU}(5) \times \mathrm{SU}(2)_{s}$ quantum numbers of the chiral superfield content of the $4 \rightsquigarrow 3$ model. The vector-like pair at the bottom of table 1a can be decoupled, resulting in a separate $4 \rightsquigarrow 3$ model.

becomes

$$
\mathscr{W}=y_{1} T \bar{F}^{\prime} \bar{F}^{\prime}+y_{2} F \bar{F}^{\prime} \phi+m F \bar{F} .
$$

Note that the additional mass term and $y_{1} \neq y_{2}$ explicitly break the $\mathrm{SU}(6)$ symmetry. Neither $F$ nor $\bar{F}$ are charged under $\mathrm{SU}(2)_{s}$, thus the confined spectrum of the model (table 1b) does not change. In the IR, the superpotential becomes

$$
\mathscr{W}=\overline{\mathcal{T}} \overline{\mathcal{T}} \overline{\mathcal{F}}+c_{1} \Lambda T \overline{\mathcal{T}}+c_{2} \Lambda F \overline{\mathcal{F}}+m F \bar{F}
$$

where the first term is the $s$-confining superpotential eq. (2.2). A simple analysis shows that in the presence of the mass term the model possesses a quantum moduli space satisfying the condition

$$
c_{2} \Lambda \overline{\mathcal{F}}+m \bar{F}=0 .
$$

While at a generic point on the moduli space the chiral SU(5) symmetry is broken, the $s$-confining vacuum where one generation acquires a mass survives at $\overline{\mathcal{F}}=\bar{F}=0$. This leaves three light generations, two made up entirely of elementary fields and another where the $\overline{\mathbf{5}}$ is made up of a linear combination of $\overline{\mathcal{F}}$ and $\bar{F}$. This lays out two interesting limits. In the limit $m \rightarrow 0$, the light generations are entirely composed of elementary fields, $\overline{\mathcal{F}}=0$, and the chirally symmetric vacuum is stabilized as in section 2.2. We refer to this as the RT limit because all composite fields decouple. In the limit $m \rightarrow \infty$, one of the three light generations has a composite $\overline{\mathbf{5}}$. We refer to this limit as the NS limit due to the appearance of light composite fields. At finite mass, there is a flat direction which can be parameterized by $\overline{\mathcal{F}}$. For the purposes of phenomenology, $\overline{\mathcal{F}}$ would play the role of a SM multiplet; motion along the moduli space of this model corresponds to motion along $D$-flat directions of a GUT (or the SM).

\section{$3.22 \rightsquigarrow 3$ generation flow}

The NS limit of the model discussed above resulted in a theory with a composite $\overline{\mathbf{5}}$ while the number of 10's (i.e. number of generations) was smaller in the IR. On the other 


\begin{tabular}{ccl}
\hline$\#$ & irrep & label \\
\hline 2 & $(\mathbf{1 0}, \mathbf{1})$ & $T$ \\
4 & $(\overline{\mathbf{5}}, \mathbf{1})$ & $\bar{F}$ \\
\hline 1 & $(\mathbf{5}, \mathbf{2})$ & $F^{\prime}$ \\
1 & $(\mathbf{1}, \mathbf{2})$ & $\phi$ \\
\hline
\end{tabular}

(a) Unconfined spectrum.

\begin{tabular}{ccl}
\hline$\#$ & irrep & label \\
\hline 3 & $(\mathbf{1 0}, \mathbf{1})$ & $T, \mathcal{T}$ \\
3 & $(\overline{\mathbf{5}}, \mathbf{1})$ & $\bar{F}$ \\
\hline 1 & $(\overline{\mathbf{5}}, \mathbf{1})$ & $\bar{F}$ \\
1 & $(\mathbf{5}, \mathbf{1})$ & $\mathcal{F}$ \\
\hline
\end{tabular}

(b) Confined spectrum.

Table 2. Summary of the $\mathrm{SU}(5) \times \mathrm{SU}(2)_{s}$ quantum numbers of the chiral superfield content of the $2 \rightsquigarrow 3$ model.

hand, original models of $[4,5]$ had a composite $\mathbf{1 0}$ in the IR thus increasing the number of generations. That construction can be interpreted as an upward generation flow. Let us discuss a variation of that model where the starting point of RG flow contains two chiral generations while the end point in the IR has three chiral generations, i.e. a $2 \rightsquigarrow 3$ model.

Once again we consider a model with the symmetry group $\mathrm{SU}(5) \times \mathrm{SU}(2)_{s}$, whose matter content and charges are given in table 2a. The tree-level superpotential in terms of the UV degrees of freedom is

$$
\mathscr{W}=y \bar{F} F^{\prime} \phi .
$$

When the non-perturbative dynamics is included, the IR superpotential becomes

$$
\mathscr{W}=\mathcal{T} \mathcal{T} \mathcal{F}+c \Lambda \bar{F} \mathcal{F},
$$

where $\mathcal{T} \sim F^{\prime} F^{\prime} / \Lambda$ and $\mathcal{F} \sim F^{\prime} \phi / \Lambda$.

It is convenient to analyze the behavior of this superpotential by going along a flat direction parameterized by $\bar{F}$. Without loss of generality we can assume that the VEV of $\bar{F}$ lives in a single component, say $\bar{F}_{5}$. At large $\mathrm{VEV}$, the global symmetry is broken from $\mathrm{SU}(5)$ to $\mathrm{SU}(4)$, and one pair of doublets, the one corresponding to the $\mathcal{F}_{5}$ meson, becomes heavy and can be integrated out. Along this flat direction the superpotential becomes

$$
\mathscr{W}=\mathcal{F}_{5}\left(\operatorname{Pf}^{\prime} \mathcal{T}+\bar{F}_{5}\right),
$$

where prime on the Pfaffian indicates that it is taken only over the light mesons comprising a 6-plet of the remaining $\mathrm{SU}(4)$ symmetry. Note that at this stage $\mathcal{F}_{5}$ is not a dynamical field since it is a meson made out of heavy doublets. At the same time, the $\bar{F}_{5}$ VEV remains arbitrary albeit related to the $\mathcal{T}$ VEVs by the $\mathcal{F}_{5}$ equation of motion,

$$
\mathrm{Pf}^{\prime} \mathcal{T}+\bar{F}_{5}=0
$$

Upon a careful inspection of (3.7) and (3.8), one notices that they correspond to the superpotential and one of the equations of motion of a four-doublet theory with a deformed moduli space, a dynamical scale $\Lambda_{L}^{6}=\bar{F}_{5} \Lambda^{5}$, and the meson $\mathcal{F}_{5}$ playing a role of Lagrange multiplier. We see that for each nonvanishing value of $\bar{F}_{5}$ the effective theory possesses a quantum deformed moduli space, i.e. it exhibits confinement with chiral symmetry breaking. Furthermore, the scale of chiral symmetry breaking is parameterized by $\bar{F}_{5}$. While the 
effective description in terms of four-doublet theory is only valid at large $\bar{F}_{5}$, the solution of the $\mathcal{F}_{5}$ equation of motion is valid everywhere on the quantum moduli space up to a $\mathrm{SU}(5)$ symmetry transformation. In particular, the chirally symmetric vacuum $\operatorname{Pf}^{\prime} \mathcal{T}=\bar{F}_{5}=0$ belongs to the quantum moduli space.

Note that the models introduced in this section differ in their quantum moduli spaces and their low-energy spectra. In the RT limit of the $4 \rightsquigarrow 3$ model, there is a unique, $s$ confining vacuum. All composite degrees of freedom become massive via the RT mechanism, and there are three light generations made out of the elementary fields. In the $2 \rightsquigarrow 3$ model and the NS limit of the $4 \rightsquigarrow 3$ model, there remains a quantum moduli space of vacua parameterized by the VEV of $\overline{\mathcal{F}}$ (or equivalently $\bar{F}$ ), respectively, which includes the chirally symmetric vacuum. In the $2 \rightsquigarrow 3$ model, one of the three light generations contains a composite 10, while at finite mass, the $4 \rightsquigarrow 3$ model has a $\overline{\mathbf{5}}$ which is partially composite and partially elementary.

In the following sections, we will show how these models can arise naturally in string model building, providing examples of phenomenologically viable string models which would have previously been ruled out by the tree-level analysis of the models.

\section{Generation flow in string models}

Given the possibility of generation flow discussed in sections 2 and 3, we will now turn our attention to string model building. Why can generation flow be relevant for string models? In string phenomenology, one tries to connect string theory to the real world (cf. e.g. [7]). In practice, this often amounts to searching for a string compactification which reproduces the SM in its low-energy limit. When constructing a string model, one chooses a framework, such as one of the perturbative string theories, and compactifies it down to four dimensions. The step of compactification consists of making an assumption on the geometry of compact dimensions (in principle one also must show that the emerging setup is stable, i.e. string moduli describing the size and shape of compact space are stabilized). However, attempts to build realistic models often fail already at an earlier stage because the zero-modes do not comprise the SM matter. This could mean that one has chiral exotics, or just not the right number of generations. It is the latter possibility where generation flow, as discussed in section 3, can be important. ${ }^{4}$ In practice, when determining the number of generations, one looks at the tree-level predictions. However, as discussed in sections 2 and 3, the number of generations obtained this way may differ from the true number of chiral generations in the low-energy effective theory. ${ }^{5}$ It is therefore interesting to study the question to which extent models of the type discussed earlier can be obtained from string theory.

\footnotetext{
${ }^{4}$ It is conceivable that more generally chiral exotics can be removed along the lines of section 2 (cf. [9] for an example). It will be interesting to work out the detailed conditions for this to happen.

${ }^{5}$ It is known that chirality-changing phase transitions can occur in string compactifications [10-12]. In this work we focus on generation flow that can be understood in terms of field-theoretic supersymmetric gauge dynamics with an $s$-confining $\mathrm{SU}(2)_{s}$ as in sections 2 and 3 . It will be interesting to see whether there is a deeper relation between these phenomena.
} 
It is not the purpose of the present paper to construct a fully realistic model exhibiting generation flow. Rather, we will collect evidence for the existence of such models. To keep our discussion simple, and in order to relate our findings to section 3 , we will look for $\mathrm{SU}(5)$ models rather than models with SM gauge group. However, we expect that the results carry over to models with the SM gauge group after compactification.

\subsection{Model scan}

In what follows, we focus on orbifold compactifications of the $\left(\mathrm{E}_{8} \times \mathrm{E}_{8}^{\prime}\right)$ heterotic string $[13$, 14], which can be efficiently constructed with the orbifolder [15]. We will collect evidence for the existence of globally consistent string compactifications that have either two or four generations of SM matter at tree level, but in fact have three generations in their lowenergy effective description. That is, we will present evidence for the existence of stringy versions of the $4 \rightsquigarrow 3$ and $2 \rightsquigarrow 3$ models discussed in section 3 .

The orbifolder allows us to compute a $4 \mathrm{D}$ model from certain input data, which comprises the geometry of the orbifold and the so-called gauge embedding. The latter essentially describes how the geometric operations of the 6D space-like compact dimensions act on the $\mathrm{E}_{8} \times \mathrm{E}_{8}^{\prime}$ lattice. This determines not only what the residual gauge symmetry of the model is but also the spectrum. In more detail, the orbifolder provides us with the continuous and discrete gauge symmetries after compactification as well as the chiral spectrum of the model.

By using the orbifolder, we obtained a large sample of supersymmetric heterotic orbifold models with the following properties:

- orbifold geometry $\mathbb{Z}_{2} \times \mathbb{Z}_{4}(1,1)$ (see [16] for the notation, and [17] for details of the geometry);

- 4D gauge group $\mathcal{G}_{4 \mathrm{D}} \supset \mathrm{SU}(5) \times \mathrm{SU}(2)_{s}$ (where we labeled the second factor " $s$ " to indicate that this $\mathrm{SU}(2)$ plays the same role as in our earlier discussion in sections 2 and 3$)$;

- the $\mathrm{SU}(5)$ and $\mathrm{SU}(2)_{s}$ gauge groups emerge each from a different $\mathrm{E}_{8}$ factor of the original heterotic string;

- a net number of $n \mathrm{SU}(5)$ GUT generations, with no representation $(\mathbf{1 0}, \mathbf{2})$ least one representation $(\overline{\mathbf{5}}, \mathbf{2})$ or $(\mathbf{5}, \mathbf{2})$;

- at least one "flavon" field transforming as $(\mathbf{1}, \mathbf{2})$; other fields of this type could in principle be decoupled from low energies;

- a (large) number of $\mathrm{SU}(5) \times \mathrm{SU}(2)_{s}$ singlets;

- additional non-Abelian gauge factors under which the SU(5) charged fields are singlets; and

- additional U(1) factors which can be broken along $D$-flat directions without breaking $\mathrm{SU}(5) \times \mathrm{SU}(2)_{s}$. 


\begin{tabular}{rcl}
\hline \multicolumn{3}{c}{$4 \rightsquigarrow 3$ model } \\
\hline$\#$ & irrep & label \\
\hline 4 & $(\mathbf{1 0}, \mathbf{1})$ & $T$ \\
4 & $(\overline{\mathbf{5}}, \mathbf{1})$ & $F$ \\
\hline 7 & $(\overline{\mathbf{5}}, \mathbf{1})$ & $F$ \\
9 & $(\mathbf{5}, \mathbf{1})$ & $\bar{F}$ \\
1 & $(\overline{\mathbf{5}}, \mathbf{2})$ & $\bar{F}^{\prime}$ \\
\hline 170 & $(\mathbf{1}, \mathbf{1})$ & $N$ \\
27 & $(\mathbf{1}, \mathbf{2})$ & $\phi$ \\
\hline
\end{tabular}

(a) The first block contains four chiral generations of $\mathrm{SU}(5)$ matter.

\begin{tabular}{rcl}
\hline \multicolumn{3}{c}{$2 \rightsquigarrow 3$ model } \\
\hline$\#$ & irrep & label \\
\hline 2 & $(\mathbf{1 0}, \mathbf{1})$ & $T$ \\
2 & $(\overline{\mathbf{5}}, \mathbf{1})$ & $F$ \\
\hline 10 & $(\overline{\mathbf{5}}, \mathbf{1})$ & $F$ \\
8 & $(\mathbf{5}, \mathbf{1})$ & $\bar{F}$ \\
1 & $(\mathbf{5}, \mathbf{2})$ & $F^{\prime}$ \\
\hline 240 & $(\mathbf{1}, \mathbf{1})$ & $N$ \\
41 & $(\mathbf{1}, \mathbf{2})$ & $\phi$ \\
\hline
\end{tabular}

(b) The first block represents two chiral families of an SU(5) GUT.

Table 3. Summary of the $\mathrm{SU}(5) \times \mathrm{SU}(2)_{s}$ quantum numbers of the (left-chiral) massless matter spectra of heterotic orbifold models with (a) $4 \rightsquigarrow 3$ and (b) $2 \rightsquigarrow 3 \mathrm{SU}(5)$ generation flow. These models have (a) four and (b) two chiral generations at tree level, respectively, but three chiral generations in the low-energy effective description due to $\mathrm{SU}(2)_{s}$ strong dynamics. The second (third) block of each table consists of states that are vector-like (invariant) under $\mathrm{SU}(5)$.

Our scan yielded several models in which $s$-confinement can change the number of chiral representations.

\subsection{Models}

Rather than providing the reader with an extensive survey, we focus on two sample models defined in the appendix. In more detail, we discuss

- a $4 \rightsquigarrow 3$ model (cf. table $3 \mathrm{a}$ ) in which the $4^{\text {th }}$ chiral generation acquires a mass and decouples through, and

- a $2 \rightsquigarrow 3$ model (cf. table $3 \mathrm{~b}$ ) in which the $3^{\text {rd }}$ chiral generation emerges from states that are vector-like under $\mathrm{SU}(5)$ through a variant of the RT effect, in which a chiral $\mathbf{1 0} \oplus \overline{\mathbf{5}}$ arises as a composite of $(\mathbf{5}, \mathbf{2}) \oplus(\mathbf{1}, \mathbf{2}) \oplus 2(\overline{\mathbf{5}}, \mathbf{1})$

Both models have the virtue that the $\mathrm{SU}(5)$ and $\mathrm{SU}(2)_{s}$ factors come from different $\mathrm{E}_{8}$ 's. Consequently, $\mathrm{SU}(2)_{s}$ can naturally be more strongly coupled than $\mathrm{SU}(5)$ (cf. e.g. [18]).

A stringy $4 \rightsquigarrow \mathbf{3}$ model. The model defined by the parameters provided in eq. (A.1) results in the $4 \mathrm{D}$ gauge group $\mathcal{G}_{4 \mathrm{D}}=\mathrm{SU}(5) \times \mathrm{SU}(2)_{s} \times\left[\mathrm{SU}(2)^{5} \times \mathrm{U}(1)^{6}\right]$. The gauge factors in the brackets can be broken along $D$-flat directions. Since the Lagrange density is invariant under complexified gauge transformation, we can infer that nontrivial solutions to the $F$-term equations preserve supersymmetry $[19,20]$. We are then left with $\mathcal{G}_{\text {unbroken }}=$ $\mathrm{SU}(5) \times \mathrm{SU}(2)_{s}$.

Before discussing the $4 \rightsquigarrow 3$ properties of this model, let us comment on the possibility to break $\mathrm{SU}(2)_{s}$ along $D$-flat directions. In this case, we will obtain a vacuum with 4 
generations of an SU(5) GUT, i.e. 4 copies of $\mathbf{1 0} \oplus \overline{\mathbf{5}}$ while the other states are now vectorlike and pick up masses proportional to the VEVs of the SU(5) singlets that got switched on. According to the usual string phenomenology practices, we would thus label this model an unrealistic 4-generation model, not worth being considered further.

On the other hand, if we leave $\mathrm{SU}(2)_{s}$ unbroken, in a generic vacuum we obtain in an intermediate step a model with 4 copies of $(\mathbf{1 0}, \mathbf{1}), 2$ copies of $(\overline{\mathbf{5}}, \mathbf{1})$, a $(\overline{\mathbf{5}}, \mathbf{2})$ and a $(\mathbf{1}, \mathbf{2})$. Since string selection rules do not forbid the corresponding couplings, the other states of table $3 \mathrm{a}$ acquire masses proportional to the VEVs of the $\mathrm{SU}(5) \times \mathrm{SU}(2) s$ singlets. Conceivably, there also exist special string vacua that can allow for an extra massless vectorlike pair $(\mathbf{5}, \mathbf{1}) \oplus(\overline{\mathbf{5}}, \mathbf{1})$. This brings us to either of the $4 \rightsquigarrow 3$ models discussed in section 3 , and summarized in table 1a. As we have seen there, due to the $\mathrm{SU}(2)_{s}$ strong dynamics, $(\overline{\mathbf{5}}, \mathbf{2})$ and $(\mathbf{1}, \mathbf{2})$ condense together to build a $\overline{\mathbf{5}}$ and condensates of $(\overline{\mathbf{5}}, \mathbf{2})$ yield an SU(5) antigeneration $\overline{\mathbf{1 0}}$. Since there are no string selection rules prohibiting the couplings, we thus expect this antigeneration to pair up with a linear combination of the 4 generations, and we are left with a 3 -generation model at low energies.

An important condition for the strong $\mathrm{SU}(2)_{s}$ dynamics to play out as described is that $\mathrm{SU}(2)_{s}$ is much more strongly coupled than $\mathrm{SU}(5)$. Since these two gauge factors originate from different $\mathrm{E}_{8}$ 's, it is plausible that this happens [18, 21, 22]. However, a detailed computation of the string thresholds is beyond the scope of this study.

A stringy $\mathbf{2} \rightsquigarrow \mathbf{3}$ model. The model defined by the parameters provided in eq. (A.2) results in the $4 \mathrm{D}$ gauge group $\mathcal{G}_{4 \mathrm{D}}=\mathrm{SU}(5) \times \mathrm{SU}(2)_{s} \times\left[\mathrm{SU}(2)^{2} \times \mathrm{U}(1)^{9}\right]$. As in the previous model, the gauge factors in parentheses can be spontaneously broken along $D$-flat directions while preserving supersymmetry. The corresponding massless spectrum after compactification is summarized in table $3 \mathrm{~b}$, where we only display the quantum numbers with respect to $\mathrm{SU}(5) \times \mathrm{SU}(2)_{s}$. After switching on the $\mathrm{VEVs}$ of $\mathrm{SU}(5) \times \mathrm{SU}(2)_{s}$ singlets, we are left with 2 copies of $(\mathbf{1 0}, \mathbf{1}), 4$ copies of $(\overline{\mathbf{5}}, \mathbf{1})$, and 1 instance of $(\mathbf{5}, \mathbf{2})$ and $(\mathbf{1}, \mathbf{2})$, reproducing the spectrum of the $2 \rightsquigarrow 3$ model presented in table 2 a.

If we also break $\mathrm{SU}(2)_{s}$ along $D$-flat directions, we obtain a vacuum with an $\mathrm{SU}(5)$ GUT symmetry and two generations of $\mathbf{1 0} \oplus \overline{\mathbf{5}}$. In the traditional approach, we would thus label the model as an unrealistic 2-generation model that is to be discarded.

However, this conclusion changes if we look at vacua where $\mathrm{SU}(2)_{s}$ confines. In this case, according to our discussion of the $2 \rightsquigarrow 3$ model in section 3 , we can obtain a third generation from $\mathrm{SU}(2)_{s}$ strong dynamics. In particular, the $(\mathbf{5}, \mathbf{2})$ builds a condensate that behaves as the 10-plet of a third generation of an SU(5) GUT. This means that this model admits 3 -generation vacua and cannot be ruled out immediately.

\subsection{Discussion}

The examples discussed in this section represent evidence for the existence of globally consistent string models with generation flow. In order to keep the discussion simple, we have focused on SU(5) models. However, we expect that qualitatively similar models with the SM gauge symmetry and matter content at low energies exist. We have verified that one can break extra gauge factors and decouple exotics by switching on VEVs along $D$-flat 
directions. We are thus guaranteed $[19,20]$ that there are supersymmetric configurations that have the features we describe. While we did verify that there are no symmetries prohibiting the required couplings, we did not compute their coefficients, nor did we explicitly verify that all directions/moduli are stabilized.

Our findings lead to the following picture. In string models, one can readily count the net number of generations at the tree-level. However, some models may have vacua where the true number of chiral generations differs from the tree-level prediction. This means that model scans in the past may have missed interesting, possibly realistic models. It will be interesting to study such constructions in more detail.

As a side remark, let us note that the matter content as well as the gauge and continuous symmetries of the RT-like model discussed in section 2 fit into a 27-plet of $\mathrm{E}_{6}$. This is evident from the branching (cf. e.g. [23])

$$
\begin{aligned}
\mathrm{E}_{6} & \rightarrow \mathrm{SU}(6) \times \mathrm{SU}(2)_{s} \\
& \rightarrow \mathrm{SU}(5) \times \mathrm{SU}(2)_{s} \times \mathrm{U}(1) \\
\mathbf{2 7} & \rightarrow(\overline{\mathbf{6}}, \mathbf{2}) \oplus(\mathbf{1 5}, \mathbf{1}) \\
& \rightarrow(\overline{\mathbf{5}}, \mathbf{2})_{1} \oplus(\mathbf{1}, \mathbf{2})_{-5} \oplus(\mathbf{1 0}, \mathbf{1})_{-2} \oplus(\mathbf{5}, \mathbf{1})_{4} .
\end{aligned}
$$

That is, while the representation content of the model may at first sight look a bit peculiar, it turns out to fit in a single chiral representation of an exceptional group. In fact, $\mathrm{E}_{6}$ is the only exceptional group admitting complex representations, and the 27-plet is its smallest representation. From this perspective it is not too surprising that variants of this model can be obtained from string theory. Note, however, that in the models which we presented, $\mathrm{SU}(5)$ and $\mathrm{SU}(2)_{s}$ stem from different $\mathrm{E}_{8}$ groups, which favors the possibility that $\mathrm{SU}(2)_{s}$ becomes strongly coupled while SU(5) does not.

\section{Summary}

We have studied the effects of non-perturbative $s$-confining dynamics on the effective number of chiral generations in supersymmetric models of particle physics. We emphasized that this number can flow either upward or downward because confinement may result in the appearance of chiral composites. In turn, these composites may either serve as new light chiral generations or lift existing chiral generations by partnering with other chiral fields in mass terms. We referred to these phenomena as generation flow.

Our focus was on $4 \rightsquigarrow 3$ and $2 \rightsquigarrow 3$ generation flow, such that in the IR there are three generations of (a GUT completion of) the SM. We analyzed the non-perturbative dynamics and verified that in our models the $s$-confining vacuum is not destabilized by the non-perturbative dynamics driving the generation flow. We stress that this conclusion is model dependent.

As we have shown, there is strong evidence that generation flow arises in globally consistent string compactifications. In particular, we have constructed explicit $4 \rightsquigarrow 3$ and $2 \rightsquigarrow 3$ models resulting from orbifold compactifications of the heterotic string. Therefore, more care than previously appreciated has to be taken when scanning for realistic string 
models. There can be models which appear to yield an unrealistic number of generations but are saved by generation flow. Furthermore, the strong dynamics that reduces the number of generations may be exploited to decouple chiral exotics of string models. Hence, the phenomenological viability of string compactifications with such exotics should be further investigated.

\section{Acknowledgments}

We would like to thank Eric Sharpe for pointing out references $[10,11]$ to us. The work of M.R., Y.S., S.S. and M.W. was supported by the National Science Foundation, under Grant No. PHY-1915005. The work of S.R.-S., M.R. and S.S. was supported by UC-MEXUSCONACyT grant No. CN-20-38. This work was performed in part at Aspen Center for Physics, which is supported by National Science Foundation grant PHY-1607611.

\section{A Orbifold model definitions}

In the bosonic formulation, a $\mathbb{Z}_{2} \times \mathbb{Z}_{4}(1,1)$ heterotic orbifold compactification is defined by the shifts $V_{1}$ and $V_{2}$ of order 2 and 4 , respectively, as well as six discrete Wilson lines $W_{a}$, $a=1, \ldots, 6$ of order 2 . These Wilson lines are restricted to satisfy $W_{1}=W_{2}$ and $W_{5}=W_{6}$ to be compatible with the $\mathbb{Z}_{2} \times \mathbb{Z}_{4}$ point group of the compactification. ${ }^{6}$ These parameters can be used as input in the orbifolder [15] to obtain the corresponding massless spectrum and compute the superpotential of the associated low-energy effective field theory.

\section{A.1 Details of the $4 \rightsquigarrow 3$ heterotic orbifold model}

One heterotic orbifold model with geometry $\mathbb{Z}_{2} \times \mathbb{Z}_{4}(1,1)$ which yields $4 \rightsquigarrow 3$ generations via the $\mathrm{RT}$ scheme is defined by the following shifts and Wilson lines (with $W_{4}=0$ ):

$$
\begin{aligned}
V_{1} & =\left(-\frac{7}{4},-\frac{1}{4},-\frac{1}{4},-\frac{1}{4},-\frac{1}{4},-\frac{1}{4}, \frac{1}{4}, \frac{7}{4}\right),(0,0,0,0,0,0,0,0), \\
V_{2} & =\left(\frac{3}{8}, \frac{1}{8}, \frac{1}{8}, \frac{1}{8}, \frac{3}{8}, \frac{9}{8},-\frac{3}{8},-\frac{3}{8}\right),\left(-1,0,0,0, \frac{1}{4}, \frac{1}{4}, \frac{1}{4}, \frac{3}{4}\right), \\
W_{1} & =W_{2}=(0,0,0,0,0,0,0,0),\left(-1,-1, \frac{1}{2}, \frac{3}{2},-\frac{1}{2}, 0, \frac{1}{2}, 0\right), \\
W_{3} & =(0,0,0,0,0,0,0,0),\left(-\frac{5}{4},-\frac{5}{4}, \frac{1}{4}, \frac{3}{4}, \frac{3}{4}, \frac{7}{4},-\frac{3}{4}, \frac{7}{4}\right), \\
W_{5} & =W_{6}=\left(-1,-1,0,1, \frac{3}{2}, \frac{1}{2}, \frac{1}{2}, \frac{3}{2}\right),(0,0,0,0,0,0,0,0) .
\end{aligned}
$$

The effective massless matter spectrum before decoupling of vector-like representations and $\mathrm{SU}(2)_{s}$ confinement, obtained by the orbifolder is summarized in table 3a.

\footnotetext{
${ }^{6}$ See e.g. [24-26] for reviews on orbifold compactifications, and [17, section 4] for more details on this specific orbifold geometry.
} 


\section{A.2 Details of the $2 \rightsquigarrow 3$ heterotic orbifold model}

The orbifold parameters that define the $\mathbb{Z}_{2} \times \mathbb{Z}_{4}(1,1)$ heterotic orbifold model presented in section 4.2 are

$$
\begin{aligned}
V_{1} & =\left(-\frac{1}{4},-\frac{1}{4}, \frac{1}{4}, \frac{1}{4}, \frac{1}{4}, \frac{1}{4}, \frac{1}{4}, \frac{9}{4}\right),(0,0,0,0,0,0,0,2), \\
V_{2} & =\left(\frac{1}{8}, \frac{9}{8},-\frac{7}{8},-\frac{1}{8},-\frac{1}{8},-\frac{1}{8}, \frac{9}{8}, \frac{7}{8}\right),\left(-\frac{1}{2}, 0,0,0, \frac{1}{4}, \frac{1}{4}, \frac{3}{4},-\frac{3}{4}\right), \\
W_{1} & =W_{2}=(1,0,-2,-1,0,1,-1,-2),\left(\frac{1}{4},-\frac{3}{4},-\frac{1}{4}, \frac{7}{4},-\frac{3}{4}, \frac{3}{4},-\frac{5}{4}, \frac{5}{4}\right), \\
W_{3} & =\left(-\frac{5}{4}, \frac{5}{4}, \frac{5}{4},-\frac{7}{4},-\frac{5}{4},-\frac{5}{4}, \frac{1}{4},-\frac{5}{4}\right),\left(\frac{7}{4}, \frac{5}{4}, \frac{7}{4}, \frac{7}{4}, \frac{5}{4}, \frac{9}{4},-\frac{1}{4}, \frac{9}{4}\right), \\
W_{5} & =W_{6}=\left(-2,-\frac{1}{2}, 0,1,-\frac{1}{2}, 1, \frac{1}{2}, \frac{3}{2}\right),\left(-\frac{7}{4},-\frac{1}{4},-\frac{5}{4},-\frac{5}{4}, \frac{7}{4}, \frac{1}{4},-\frac{3}{4},-\frac{7}{4}\right),
\end{aligned}
$$

and $W_{4}=0$. Using these parameters as input of the orbifolder, one finds the massless matter spectrum before decoupling of vector-like representations and $\mathrm{SU}(2)_{s}$ confinement shown in table $3 \mathrm{~b}$.

Open Access. This article is distributed under the terms of the Creative Commons Attribution License (CC-BY 4.0), which permits any use, distribution and reproduction in any medium, provided the original author(s) and source are credited.

\section{References}

[1] N. Seiberg, Exact results on the space of vacua of four-dimensional SUSY gauge theories, Phys. Rev. D 49 (1994) 6857 [hep-th/9402044] [INSPIRE].

[2] C. Csáki, M. Schmaltz and W. Skiba, A Systematic approach to confinement in $N=1$ supersymmetric gauge theories, Phys. Rev. Lett. 78 (1997) 799 [hep-th/9610139] [INSPIRE].

[3] C. Csáki, M. Schmaltz and W. Skiba, Confinement in $N=1$ SUSY gauge theories and model building tools, Phys. Rev. D 55 (1997) 7840 [hep-th/9612207] [INSPIRE].

[4] M.J. Strassler, Generating a fermion mass hierarchy in a composite supersymmetric standard model, Phys. Lett. B 376 (1996) 119 [hep-ph/9510342] [INSPIRE].

[5] A.E. Nelson and M.J. Strassler, A Realistic supersymmetric model with composite quarks, Phys. Rev. D 56 (1997) 4226 [hep-ph/9607362] [InSPIRE].

[6] S.S. Razamat and D. Tong, Gapped Chiral Fermions, Phys. Rev. X 11 (2021) 011063 [arXiv:2009.05037] [INSPIRE].

[7] L.E. Ibáñez and A.M. Uranga, String theory and particle physics: An introduction to string phenomenology, Cambridge University Press (2012) [DOI].

[8] S. Ramos-Sánchez, M. Ratz, Y. Shirman, S. Shukla and M. Waterbury, in preparation.

[9] P. Dimopoulos, G.K. Leontaris and N.D. Tracas, Supercompositeness inspired from superstrings, Z. Phys. C 76 (1997) 327 [hep-ph/9604265] [INSPIRE].

[10] S. Kachru and E. Silverstein, Chirality changing phase transitions in 4-D string vacua, Nucl. Phys. B 504 (1997) 272 [hep-th/9704185] [INSPIRE]. 
[11] M.R. Douglas and C.-g. Zhou, Chirality change in string theory, JHEP 06 (2004) 014 [hep-th/0403018] [INSPIRE].

[12] L.B. Anderson, J. Gray, N. Raghuram and W. Taylor, Matter in transition, JHEP 04 (2016) 080 [arXiv: 1512.05791] [INSPIRE].

[13] L.J. Dixon, J.A. Harvey, C. Vafa and E. Witten, Strings on Orbifolds, Nucl. Phys. B 261 (1985) 678 [INSPIRE].

[14] L.J. Dixon, J.A. Harvey, C. Vafa and E. Witten, Strings on Orbifolds. 2, Nucl. Phys. B 274 (1986) 285 [INSPIRE].

[15] H.P. Nilles, S. Ramos-Sanchez, P.K.S. Vaudrevange and A. Wingerter, The Orbifolder: A Tool to study the Low Energy Effective Theory of Heterotic Orbifolds, Comput. Phys. Commun. 183 (2012) 1363 [arXiv:1110.5229] [INSPIRE].

[16] M. Fischer, M. Ratz, J. Torrado and P.K.S. Vaudrevange, Classification of symmetric toroidal orbifolds, JHEP 01 (2013) 084 [arXiv:1209.3906] [INSPIRE].

[17] D.K. Mayorga Pena, H.P. Nilles and P.-K. Oehlmann, A Zip-code for Quarks, Leptons and Higgs Bosons, JHEP 12 (2012) 024 [arXiv:1209.6041] [INSPIRE].

[18] L.E. Ibáñez and H.P. Nilles, Low-Energy Remnants of Superstring Anomaly Cancellation Terms, Phys. Lett. B 169 (1986) 354 [InSPIRE].

[19] F. Buccella, J.P. Derendinger, S. Ferrara and C.A. Savoy, Patterns of Symmetry Breaking in Supersymmetric Gauge Theories, Phys. Lett. B 115 (1982) 375 [INSPIRE].

[20] M.A. Luty and W. Taylor, Varieties of vacua in classical supersymmetric gauge theories, Phys. Rev. D 53 (1996) 3399 [hep-th/9506098] [inSPIRE].

[21] L.J. Dixon, V. Kaplunovsky and J. Louis, Moduli dependence of string loop corrections to gauge coupling constants, Nucl. Phys. B 355 (1991) 649 [INSPIRE].

[22] S. Stieberger, $(0,2)$ heterotic gauge couplings and their M-theory origin, Nucl. Phys. B $\mathbf{5 4 1}$ (1999) 109 [hep-th/9807124] [INSPIRE].

[23] R. Slansky, Group Theory for Unified Model Building, Phys. Rept. 79 (1981) 1 [InSPIRE].

[24] D. Bailin and A. Love, Orbifold compactifications of string theory, Phys. Rept. 315 (1999) 285.

[25] S. Ramos-Sanchez, Towards Low Energy Physics from the Heterotic String, Fortsch. Phys. 10 (2009) 907 [arXiv: 0812.3560] [INSPIRE].

[26] P.K.S. Vaudrevange, Grand Unification in the Heterotic Brane World, Ph.D. thesis, Bonn University (2008) arXiv:0812.3503 [INSPIRE]. 